\title{
Botany
}

American Journal of Botany: e176-e178. 2012.

aJB Primer Notes \& Protocols in the Plant Sciences

\section{Microsatellite MARKERS ISOLATED FROM THE WILD MEdicinal Plant Centella asiatica (APIACEAe) From an ENRICHED GENOMIC LIBRARY ${ }^{1}$}

\author{
Soaharin'ny Ony Raoseta Rakotondralambo ${ }^{2,3}$, Alexandra Lussert ${ }^{4}$, Ronan Rivallan $^{4}$, \\ Pascal Danthu ${ }^{3,5}$, Jean-Louis Noyer ${ }^{4}$, and Franc-Christophe Baurens ${ }^{4,6}$
}

\begin{abstract}
${ }^{2}$ Département de Biologie et Ecologie végétales, Université d'Antananarivo, BP 906 Antananarivo, Madagascar; ${ }^{3}$ CIRAD, URP Forêts et Biodiversité, BP 853, Antananarivo, Madagascar; ${ }^{4}$ CIRAD, UMR AGAP 34098 Montpellier France; and ${ }^{5}$ CIRAD, UR 10534398 Montpellier, France
\end{abstract}

- Premise of the study: Microsatellite markers for Centella asiatica, an important medicinal herb, were developed and characterized to promote genetic and molecular studies.

- Methods and Results: A GA/GT-enriched genomic library was constructed from an accession from Madagascar. Roughly $75 \%$ of the 768 clones of the enriched library contained microsatellites. Eighty sequences containing microsatellites were obtained from 96 positive clones. Specific primers were designed for 20 loci, and 17 of them displayed polymorphism when screened across 17 C. asiatica accessions, with an average of 4.3 alleles per locus. The observed and expected heterozygosity values averaged 0.114 and 0.379 , respectively.

- Conclusions: This is the first report constructing an enriched genomic library and identifying microsatellite markers from C. asiatica. These 17 polymorphic microsatellite markers are a useful resource for this plant, applicable for diversity studies, pedigree analyses, and genetic mapping.

Key words: Apiaceae; Centella asiatica; enriched genomic library; Madagascar; microsatellites; simple sequence repeat markers.

Centella asiatica (L.) Urban (Apiaceae) is an important medicinal herb that is used in both traditional pharmacopoeia and modern medicine. Asiaticoside is one of the principal compounds commercially used as a wound-healing agent, owing primarily to its anti-inflammatory effects. Madecassol and Blastoestimulina are the most notable pharmaceutical products derived from this plant (Randriamampionona et al., 2007). Centella asiatica is a perennial, herbaceous creeper, mainly found in tropical and subtropical countries. Madagascar is the world's top C. asiatica producer, and the species is second only to Madagascar periwinkle (Catharanthus roseus) as a medicinal plant export (Péchard et al., 2005). Chemical, medicinal, and pharmacological studies have been conducted to better understand the medicinal properties of pentacyclic triterpenoids from $C$. asiatica (Pittella et al., 2009); however, little is known about its biology and genetics. This plant has been reported to be diploid with $2 n=2 x=18$, but some older controversial studies questioned either the number of

${ }^{1}$ Manuscript received 6 September 2011; revision accepted 2 January 2012.

The authors would like to thank the Montpellier-Languedoc Roussillon Grand Plateau Technique Regional for hosting SSR genotyping; the French Government and CIRAD for allocated scholarships; the French Ministry of Foreign Affairs (FSP/FORMA, PARRUR), the European Union (FOREAIM), and Yves Rocher group for financial assistance; Indena S.A, Italy, for kindly donating Centella asiatica India samples; G. Oliver for technical assistance in greenhouses; A. M. Risterrucci and B. Courtois for fruitful discussion on the manuscript; and K. Newby for manuscript correction.

${ }^{6}$ Author for correspondence: franc-christophe.baurens@cirad.fr

doi:10.3732/ajb.1100441 chromosomes, the presence of B chromosomes, or ploidy level (Das and Mallick, 1991). Sequence data are restricted to some expressed sequence tags, mRNA, and PCR-amplified gene fragments, which have been used for phylogenetic analysis of the Apiaceae (Downie et al., 2000). In this paper, we report the development and characterization of microsatellite markers for $C$. asiatica that can be used for taxonomic, diversity, and phylogenetic studies of this species.

\section{METHODS AND RESULTS}

An accession from Vohimana (Appendix 1), in eastern Madagascar, was used to construct a microsatellite-enriched library following the protocol described by Billotte et al. (1999). Ten micrograms of genomic DNA were digested with RsaI endonuclease and ligated to RSA21 and RSA25 adapters. Ten nanograms of ligated DNA were amplified with RSA21 primer and purified. The PCR products were submitted to a hybridization-based capture with $5^{\prime}$ biotine-labeled microsatellite oligoprobes $\mathrm{I} 5(\mathrm{GA})_{8}$ and $\mathrm{I} 5(\mathrm{GT})_{8}$ using streptavidine-coated magnetic beads (Promega, Madison,Wisconsin, USA). The eluted fraction was PCR amplified with RSA21 primer, cloned into pGEM-T Easy vector (Promega), and then transformed into DH10BT1R Escherichia coli strain (Invitrogen, Carlsbad, California, USA). Inserts from white colonies were PCR amplified, separated onto $1.2 \%$ agarose gel, transferred onto Hybond-N+ nylon membranes (GE Healthcare, Fairfield, Connecticut, USA), and hybridized at $56^{\circ} \mathrm{C}$ with $[\gamma 32 \mathrm{P}]$ ATP $5^{\prime}$ end-labeled $(\mathrm{GA})_{15}$ and $(\mathrm{GT})_{15}$ probes.

Among 768 clones of the microsatellite-enriched genomic library of $C$. asiatica, 560 contained simple sequence repeat (SSR) markers, giving an enrichment rate of $73 \%$. Ninety-six of these clones were selected based on the strength of hybridization signal and sequenced using a 96-capillary 3730xl DNA analyzer (Applied Biosystems, Foster City, California, USA) at GATC Biotech Company (Konstanz, Germany). We analyzed the sequences and designed primer 
pairs with the dedicated pipeline SAT (Dereeper et al., 2007), using default parameters proposed for enriched libraries. We obtained 76 singletons and four clusters with more than two sequences. Thirty-seven sequences $(46.25 \%)$ contained compound dinucleotide motifs (i.e., combination of AG, GT, and AT motifs), 32 sequences (40\%) harbored simple dinucleotide repetitions, and 11 sequences $(13.75 \%)$ harbored compound complex di-, tri-, tetra-, hexa-, and pentanucleotide motifs. Primer pairs were designed for 30 SSR markers. All gave amplification products on the reference plant DNA and also on DNA extracted from the putative closely related species $C$. uniflora. Only 20 SSR markers were repeatable and easy to score, and were conserved for further polymorphism study.

To assess microsatellite polymorphism, DNA from 17 accessions was used: 16 C. asiatica accessions collected in Madagascar and one accession from India (see Appendix 1 for precise locations). Characteristics of the 20 SSR markers are shown in Table 1. PCR amplifications were performed in a final volume of $10 \mu \mathrm{L}$ containing $25 \mathrm{ng}$ of DNA as template, following the PCR protocol of Oblessuc et al. (2009) and resolved on an ABI3500xl DNA sequencer (Applied Biosystems). Seventeen markers were polymorphic and subsequently analyzed; the remaining three were monomorphic. A total of 73 alleles were produced at the 17 polymorphic loci, with an average of 4.3 alleles per locus. Four SSR markers (mCaCIR004, mCaCIR009, mCaCIR019, mCaCIR024) showed low polymorphism with two alleles per locus, and the rest were polymorphic with more than four alleles per locus (Table 2). No difference of polymorphism level could be observed between SSRs derived from pure dinucleotide, compound, or complex motifs. Genetic analysis was carried out using PowerMarker software 3.25 (Liu and Muse, 2005). Observed heterozygosity $\left(H_{\mathrm{o}}\right)$ and expected heterozygosity $\left(H_{\mathrm{e}}\right)$ were calculated according to the formula of Nei (1973); mean $H_{\mathrm{o}}$ was 0.114 (range: 0-0.625) and mean $H_{\mathrm{e}}$ was 0.379 (range: $0.218-0.585$ ) (Table 2). Significant linkage disequilibrium was found for 114 marker pairs of the 136 possible pairs.

\section{CONCLUSIONS}

The values obtained for $H_{\mathrm{o}}$ and $H_{\mathrm{e}}$ correspond to SSR loci with a medium level of polymorphism. Discrepancy between $H_{\mathrm{o}}$ and $H_{\mathrm{e}}$ could be explained by null alleles, inbreeding, or by the small sample size. Similarly, the significant linkage disequilibrium reported here might reflect either significant genetic differentiation among populations of $C$. asiatica or strong genetic linkage between markers. Nevertheless, taking into account the small size of the sample, more data are required to confirm the cause of linkage disequilibrium, and new insight into the biology of $C$. asiatica is needed to clarify

TABLE 1. Characterization of 17 microsatellites in Centella asiatica.

\begin{tabular}{|c|c|c|c|c|c|}
\hline SSR locus & Primer sequences $\left(5^{\prime}-3^{\prime}\right)$ & Repeat motif & Allele size range (bp) & $T_{\mathrm{a}}\left({ }^{\circ} \mathrm{C}\right)$ & EMBL accession no. \\
\hline mCaCIR002 & F: CCACAGGTAACACCGAAT & $(\mathrm{CT})_{30}$ & $141-169$ & 55 & FR838931 \\
\hline mCaCIR004 & $\begin{array}{l}\text { R: GCACTTGCACTATCTGGAA } \\
\text { F: GGGTGGTCTGCCTAAAGA }\end{array}$ & (CT) & 100202 & 56 & \\
\hline 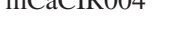 & $\begin{array}{l}\text { F: GGGIGGICIGCCIAAAGA } \\
\text { R: TGGAGATCAAGTTCATGC }\end{array}$ & $(\mathrm{Cl})_{17}$ & $190-202$ & 56 & FR838932 \\
\hline $\mathrm{mCaCIR005*}$ & $\begin{array}{l}\text { F: GGCCTTCAATGTATGCTG } \\
\text { R: TTTGATTTGTTGGGTCTTG }\end{array}$ & $(\mathrm{GA})_{16}$ & 170 & 55 & HE605293 \\
\hline mCaCIR006 & $\begin{array}{l}\text { F: ACGGGCATTTATTCCATT } \\
\text { R: GCAAACCACCACAACTTC }\end{array}$ & $(\mathrm{CT})_{15}$ & $215-219$ & 55 & FR838933 \\
\hline mCaCIR007 & $\begin{array}{l}\text { F: TGGAGGTGGTGTAACTGG } \\
\text { R: AGGGGATCAAACCTCATC }\end{array}$ & $(\mathrm{TG})_{12}$ & $234-240$ & 55 & FR838934 \\
\hline mCaCIR009 & $\begin{array}{l}\text { F: TGCCTATCCTTTGAATGC } \\
\text { R: CAAACATGACATTCTTAAAACA }\end{array}$ & $(\mathrm{TC})_{11}$ & $289-295$ & 55 & FR838935 \\
\hline mCaCIR010 & $\begin{array}{l}\text { F: AATGTAAAATTCCCGGTGT } \\
\text { R: TAAACAGGCGTTCCAAGT }\end{array}$ & $(\mathrm{TG})_{10}$ & $233-251$ & 54 & FR838936 \\
\hline mCaCIR011 & $\begin{array}{l}\text { F: TTCATAAAAGTCCTTCCACA } \\
\text { R: TAGGTTGATGTGGCCTCT }\end{array}$ & $(\mathrm{CA})_{10}$ & $215-217$ & 53 & FR838937 \\
\hline mCaCIR012 & $\begin{array}{l}\mathrm{F}: \text { CACGAAAATTGGAAACAA } \\
\mathrm{R}: \text { CATGTGAGTTTATGAGTTTCTATG }\end{array}$ & $(\mathrm{AC})_{10}$ & $207-217$ & 53 & FR838938 \\
\hline mCaCIR013* & $\begin{array}{l}\mathrm{F}: \text { CAAGTTCCTCCCACGAAT } \\
\mathrm{R}: \text { GCCGAAATAATCGAAATATAAG }\end{array}$ & $(\mathrm{AC})_{9}$ & 201 & 55 & HE605294 \\
\hline mCaCIR018 & $\begin{array}{l}\text { F: TTGAGTTTAAGAAGTCCCAAAT } \\
\text { R: AATCCTTCACACTCCTAAAGC }\end{array}$ & $(\mathrm{TC})_{23}(\mathrm{TA})_{7}$ & $174-221$ & 55 & FR838939 \\
\hline mCaCIR019 & $\begin{array}{l}\text { F: TTTCTTGTTAAATGCGATGA } \\
\text { R: AATGACATCACTGCTATGGA }\end{array}$ & $(\mathrm{GA})_{15}(\mathrm{GTGC})_{3}$ & $206-212$ & 54 & FR838940 \\
\hline mCaCIR020 & $\begin{array}{l}\text { F: TTTAGGAAGTTGGATTTTGC } \\
\text { R: GGTTTAATTCAGGACGCTTA }\end{array}$ & $(\mathrm{AC})_{7}(\mathrm{AC})_{5}$ & 174-194 & 55 & FR838941 \\
\hline $\mathrm{mCaCIR} 021 *$ & $\begin{array}{l}\text { F: TGCCTAGATTTTGGGTTTT } \\
\text { R: TCTTACAATGCAATCAACCT }\end{array}$ & $(\mathrm{CT})_{24}(\mathrm{ATT})_{4}$ & 200 & 55 & HE605295 \\
\hline $\mathrm{mCaCIR} 022$ & $\begin{array}{l}\text { F: AGGAGTATTGACAAGAGGTGA } \\
\text { R: GGATGGCAGTCCATTTTA }\end{array}$ & $(\mathrm{CT})_{6}(\mathrm{CT})_{11}(\mathrm{CA})_{9}$ & $245-261$ & 54 & FR838942 \\
\hline mCaCIR024 & $\begin{array}{l}\text { F: TCTTTCGTTGATACATGCAC } \\
\text { R: AAAACTTAAAGAAGATACAAACTCC }\end{array}$ & $(\mathrm{ATTA})_{3}$ & $241-275$ & 55 & FR838943 \\
\hline mCaCIR027 & $\begin{array}{l}\mathrm{F}: \text { ACCCCAAGACCTTCAGTT } \\
\mathrm{R}: \text { CCTTCTGCTTTCCCTTTT }\end{array}$ & $(\mathrm{CA})_{6}(\mathrm{AG})_{10}$ & $225-233$ & 54 & FR838944 \\
\hline mCaCIR028 & $\begin{array}{l}\text { F: CAGAGTTTGGGCAGAAAA } \\
\text { R: GACGAGTGGAGGATAAGAAA }\end{array}$ & $(\mathrm{AG})_{5}(\mathrm{AG})_{8}$ & 197-203 & 55 & FR838945 \\
\hline mCaCIR029 & $\begin{array}{l}\text { F: GGTCTGAGGTCTGTTGAGG } \\
\text { R: CGCATTGACAGAACAAAA }\end{array}$ & $(\mathrm{CA})_{8}(\mathrm{AT})_{5}(\mathrm{CT})_{5}$ & $317-366$ & 55 & FR838946 \\
\hline mCaCIR030 & $\begin{array}{l}\text { F: GGCAAATCGAGAGCAATA } \\
\text { R: ACGGAAAAGCCTAACAGC }\end{array}$ & $(\mathrm{TG})_{5}(\mathrm{TA})_{5}(\mathrm{GA})_{20}$ & $240-248$ & 55 & FR838947 \\
\hline
\end{tabular}

Note $: \mathrm{EMBL}=$ European Molecular Biology Laboratory; $T_{\mathrm{a}}=$ annealing temperature.

*Indicates monomorphic marker. 
TABLE 2. Genetic data for 17 accessions of Centella asiatica using the polymorphic microsatellite markers developed in this study.

\begin{tabular}{llll}
\hline \hline SSR locus & $N_{\mathrm{a}}$ & \multicolumn{1}{c}{$H_{\mathrm{o}}$} & $H_{\mathrm{e}}$ \\
\hline mCaCIR002 & 5 & 0 & 0.226 \\
mCaCIR004 & 3 & 0 & 0.32 \\
mCaCIR006 & 4 & 0 & 0.218 \\
mCaCIR007 & 6 & 0.063 & 0.377 \\
mCaCIR009 & 3 & 0 & 0.218 \\
mCaCIR010 & 6 & 0.625 & 0.585 \\
mCaCIR011 & 4 & 0.188 & 0.365 \\
mCaCIR012 & 4 & 0.063 & 0.279 \\
mCaCIR018 & 5 & 0.125 & 0.527 \\
mCaCIR019 & 2 & 0 & 0.226 \\
mCaCIR020 & 4 & 0.063 & 0.369 \\
mCaCIR022 & 5 & 0 & 0.5 \\
mCaCIR024 & 3 & 0.25 & 0.576 \\
mCaCIR027 & 5 & 0.188 & 0.541 \\
mCaCIR028 & 4 & 0 & 0.219 \\
mCaCIR029 & 5 & 0.25 & 0.494 \\
mCaCIR030 & 5 & 0.125 & 0.414 \\
\hline
\end{tabular}

Note: $H_{\mathrm{e}}=$ expected heterozygosity; $H_{\mathrm{o}}=$ observed heterozygosity; $N_{\mathrm{a}}=$ number of alleles.

ploidy and reproductive biology, before these SSR markers can be used for population genetic studies. Despite these potential limitations, a wide range of plant genetic studies can now be envisaged, such as diversity studies and pedigree analyses. Genetic mapping, which requires a higher number of markers, is now possible based on this microsatellite-enriched library of $C$. asiatica, which potentially contains hundreds of SSRs. Finally, the cross-species amplification observed here will allow phylogenetic analyses and clarify taxonomic status within Centella.

\section{LITERATURE CITED}

Billotte, N. B., J. M. P. Lagoda, A. M. Risterucci, and F. C. Baurens. 1999. Microsatellite enriched libraries: Applied methodology for the development of SSR markers in tropical crops. Fruits 54: 277-288.

DAS, A., AND R. MALLICK. 1991. Correlation between genomic diversity and asiaticoside content in Centella asiatica (L.) Urban. Botanical Bulletin of Academia Sinica 32: 1-8.

Dereeper, A., X. Argout, C. Billot, J. F. Rami, and M. Ruiz. 2007. SAT, a flexible and optimized web application for SSR marker development. BMC Bioinformatics 8: 465. Website http://southgreen.cirad.fr/ [accessed 9 March 2012].

Downie, S. R., D. S. Katz-Downie, and M. F. Watson. 2000. A phylogeny of the flowering plant family Apiaceae based on chloroplast DNA rpl16 and rpoC1 intron sequences: Towards a suprageneric classification of subfamily Apioideae. American Journal of Botany 87: 273-292.

Liu, K., AND S. V. MusE. 2005. PowerMarker: Integrated analysis environment for genetic marker data. Bioinformatics (Oxford, England) 21 2128-2129.

NeI, M. 1973. Analysis of gene diversity in subdivided populations. Proceedings of the National Academy of Sciences, USA 70: 3321-3323.

Oblessuc, P. O., T. Campos, J. M. K. Cardoso, D. A. Sforça, R. M. Baroni, A. P. Souza, ANd L. L. Benchimol. 2009. Adaptation of fluorescent technique for genotyping with new microsatellite markers in common bean. Pesquisa Agropecuaria Brasileira 44: 638-644.

Péchard, G., M. Antona, S. Aubert, and D. Babin. 2005. Ressources phytogénétiques, contrats et application de la Convention biodiversité à Madagascar: Une approche prospective. Bois et Forêts des Tropiques 284: 45-57.

Pittella, F., R. C. Dutra, D. D. Junior, M. T. P. Lopes, and N. R. Barbosa. 2009. Antioxidant and cytotoxic activities of Centella asiatica (L.) Urb. International Journal of Molecular Sciences 10: 3713-3721.

Randriamampionona, D., B. Diallo, F. Rakotoniriana, C. Rabemanantsoa, K. Cheuk, A. M. Corbisier, J. Mahillon, S. Ratsimamanga, and M. El JAZIRI. 2007. Comparative analysis of active constituents in Centella asiatica samples from Madagascar: Application for ex situ conservation and clonal propagation. Fitoterapia 78: 482-489.
ApPendix 1. Voucher information for the 17 accessions from Madagascar used to assess microsatellite polymorphism of Centella asiatica. Voucher specimens are deposited at Tsimbazaza Herbarium (TAN), Madagascar; five samples were collected per location.

\begin{tabular}{llrll}
\hline \hline Region & $\begin{array}{c}\text { Collection } \\
\text { location }\end{array}$ & Altitude $(\mathrm{m})$ & Longitude $\left({ }^{\circ} \mathrm{E}\right)$ & Latitude $\left({ }^{\circ} \mathrm{S}\right)$ \\
\hline Antananarivo & Ambohitromby & 803 & 46.273888 & 17.989167 \\
Antananarivo & Tsiroanomandidy & 940 & 46.059816 & 18.754022 \\
Antananarivo & Belobaka & 820 & 45.697253 & 18.993603 \\
Antananarivo & Mandoto & 1475 & 46.311173 & 19.573930 \\
Diego & Ambanja & 5 & 48.428727 & 13.721140 \\
Mahajanga & Antsohihy & 21 & 48.049614 & 14.912999 \\
Mahajanga & Besalampy I & 39 & 44.493775 & 16.746286 \\
Mahajanga & Kandreho & 199 & 46.091667 & 17.485277 \\
Mahajanga & Ambatomainty & 438 & 45.991111 & 17.416944 \\
Mahajanga & Maintirano & 26 & 44.033056 & 18.063687 \\
Mahajanga & Besara Sahoany & 55 & 44.287228 & 18.684945 \\
Mahajanga & Antsalova & 117 & 44.619426 & 18.681393 \\
Mahajanga & Tsiandro & 730 & 44.900000 & 18.730000 \\
Toamasina & Vohimana & 963 & 48.836877 & 18.957978 \\
Toliara & Miandrivazo & 154 & 45.454100 & 19.532300 \\
Toliara & Beroboka & 47 & 44.607971 & 19.966997 \\
\hline
\end{tabular}

